\title{
ARCHIVOS E INVESTIGACIÓN. REFLEXIONES EN TORNO A LAS POSIBILIDADES DE INDAGACIÓN DE LAS RELACIONES DE GÉNERO EN LOS ARCHIVOS*
}

YAMILA BALBUENA Y MARIANA NAZAR

(UNLP-CPM); yamilabalbuena2003@gmail.com

(UBA-AGN); mariananazar@gmail.com

RESUMEN

Este trabajo surge como respuesta a determinadas propuestas que se vienen dando en la Argentina en relación con el tratamiento y difusión de documentos de archivo por parte de los/as investigadores sociales.

En el mismo, nos dedicaremos a abordar una primera diferenciación entre las tareas del archivo y las tareas de la investigación, pasando luego a explicitar algunas diferencias entre los distintos reservorios de información, para culminar en un acercamiento a las formas de indagación de las relaciones de género en los archivos, no sin antes mencionar la problemática general en la que se encuentran hoy los archivos en nuestro país.

Palabras clave: archivos, perspectiva de género, investigación, archivística, nueva historia

ABSTRACT

This paper attempt to answer certain proposition about archive policies that are going on in Argentina, especially related to the dissemination and treatment of archive's documents developed by social researchers.

In order to analyse, we distinguish between researching work and the archive one's first. Then, we characterize the different types of archives. Finally, we focus on two problematic topics: the different ways of approaching to gender relationships contained in the archives's collections, and the widespreaded difficulties that archives face nowadays in our

country

Key words: Archive, gender perspective, researching, archival science, New History

\footnotetext{
* Una primera aproximación a este trabajo fue presentado como ponencia en el II Coloquio de Historia, Género y Política en los 70, organizado por el Instituto Interdisciplinario de Estudios de Género de la Facultad de Filosofía y Letras de la U.B.A. y el Museo Histórico Roca, en la Ciudad de Buenos Aires durante agosto de 2006. Agradecemos los comentarios que sobre esta presentación realizaron Rubén Dalceggio y Andrés Pak Linares.
} 
Este trabajo surge a partir de la inquietud que como historiadoras y archivistas nos generan determinadas propuestas que se vienen dando en la Argentina, en un contexto de difusión de nuevas perspectivas historiográficas y auge de trabajos referidos a los conceptos de memoria que creen ver en la creación de nuevos archivos la respuesta a las lagunas documentales que parecieran existir en referencia a sus campos de investigación.

Partiendo de la premisa de que no se debería confundir un archivo con un centro de documentación (distintos repositorios para distintas necesidades) estas lagunas no pueden cubrirse sino recuperando y jerarquizando archivos ya existentes, trabajándolos, poniéndolos a la consulta pública, en fin, democratizando su acceso.

Para ilustrar la compleja relación entre la aplicación de nuevas metodologías de investigación y el tratamiento de nuevas fuentes, trabajaremos con el caso de los estudios de género.

En este sentido nos dedicaremos a abordar una primera diferenciación entre las tareas del archivo y las tareas de la investigación, pasando luego a explicitar las diferencias entre los distintos reservorios de información, para culminar en un acercamiento a las formas de indagación de las relaciones de género en los archivos, no sin antes mencionar la problemática general en la que se encuentran hoy los archivos en nuestro país.

\section{Sobre las especificidades del archivo}

Un archivo es, por definición, la documentación producida por una institución o persona en el desarrollo de sus funciones. Un archivo es el sedimento documental de una organización o una persona, los documentos que "quedaron", por su importancia, en ese pasado. Un documento de archivo es, entonces, el testimonio de la actividad desarrollada por una persona física o jurídica, pública o privada cuyas características particulares son: originalidad, organicidad y carácter seriado. Éstas, lo distinguen del resto de los documentos; absolutamente válidos como testimonios del pasado, pero de otro orden (p.e. arquitectónico, tradición oral, museológico, bibliotecológico...). Y esto hace que los documentos de archivo no sólo tengan por finalidad ser fuentes para la historia, sino también ser garantes de derechos.

Asimismo, y desde la mirada de las posibilidades que estos brindan como fuentes para la investigación, dichas especificidades nos permiten analizarlos a partir de reconocer que no fueron 206 
producidos para su consulta hermenéutica posterior, sino con una finalidad administrativa. Es por esto que los documentos de archivo, al dar cuenta de una misión específica del organismo productor, permiten ser analizados desde distintas perspectivas, como puede ser: traer a la superficie o analizar, temáticas como las relaciones de dominación, los idearios, la ética, etc.

Las tareas que se realizan para preservar, ordenar, clasificar, describir, poner a la consulta y difundir este tipo de documentos son de un orden completamente diferente a las tareas que se realizan al investigar sobre ellos. De hecho, aunque en nuestro país no esté muy difundida ni reconocida, existe una disciplina específica para el tratamiento de los mismos: la archivística. Esta surge en forma moderna a principios del siglo XX estableciendo una metodología propia a partir de las distintas experiencias y reflexiones teóricas, y se sigue desarrollando a lo largo de todo el siglo, teniendo como institución de encuentro el Consejo Internacional de Archivos. El crecimiento de esta disciplina ha sido muy grande, especialmente desde la segunda mitad del siglo XX, cuando empezó a trabajar con los documentos desde su origen.

A pesar de la existencia de una disciplina específica (que no es reconocida por el Estado, y en general tampoco por las organizaciones de la sociedad civil para el trabajo en archivos) y debido seguramente a la grave situación por la que atraviesan los archivos en nuestro país es muy frecuente encontrar investigadores gestionando archivos.

El problema que en esto encontramos no radica en que sean investigadores, sino en las diferencias, que parecieran no estar claras, que tiene trabajar en uno u otro sentido. Considerando que la ordenación de archivos se rige por el sentido común, suelen organizar el trabajo como investigadores, no como archivistas. Los problemas más recurrentes suelen ser: la utilización arbitraria de la terminología archivística (desde la utilización indistinta del nombre de archivo para centros de documentación, bibliotecas o hemerotecas, hasta hablar de accesibilidad refiriéndose a acceso), las diversas formas de ordenamiento y descripción documental (el más frecuente en el caso de los investigadores manipulando y describiendo fondos o colecciones es la fascinación que ejerce en ellos el ordenamiento y el índice temático ${ }^{1}$ ), el desconocimiento de las medidas de preservación de los distintos soportes y, por lo tanto, los riesgos de daño irreversible al

\footnotetext{
${ }^{1}$ Esto hace que se rompa el principio de procedencia al mezclar y desmembrar fondos y colecciones y, en el caso del índice, suele no surgir de un inventario, sino que se transforma en el único auxiliar para consultar esos documentos, auxiliar que suele ser incomprensible para aquellos que no comparten la disciplina del investigador-descriptor o que no comparten su corriente de pensamiento debido a la ausencia de tesauro y que requiere de una gran inversión en tiempo de descripción sin el resultado proporcionado de una búsqueda rápida.
} 
pretender preservarlos, el establecimiento de prioridades relacionadas más con la reprografía de documentos que con la preservación y la elaboración de instrumentos de descripción de los soportes originales (o la idea de que cuando un documento ya está digitalizado no importan las condiciones en que se conserve su soporte original, ni se hace necesario realizar el inventario) y el planteo de diversas discusiones sobre cuestiones que la archivística ya ha saldado o, por lo menos, le ha dedicado varios estudios, lo cual los lleva a inventar nuevos términos.

El desconocimiento o falta de aplicación de la disciplina, tanto en el ámbito oficial como en el no oficial y el privado, tiene la misma consecuencia: la pérdida de documentos, tiempo, recursos e información.

Para ejemplificar las problemáticas relacionadas con el tratamiento de documentos de archivo, en este trabajo tomaremos como análisis de caso los estudios denominados de género ${ }^{2}$.

En este sentido, nos permitiremos reflexionar sobre las diferencias metodológicas y epistemológicas que atraviesan la creación de centros de documentación, el recuperar y poner a la consulta pública fondos documentales de organizaciones de mujeres o fondos particulares de las mismas.

\section{Sobre la perspectiva de género}

No pretendiendo realizar un estado de la cuestión sobre la problemática de género, lo que tomaremos de esta perspectiva es la relación que puede establecerse entre nuevas formas de enfocar la historia, metodologías de investigación y utilización de documentos de archivo.

Los estudios de mujeres/estudios de género se abren paso en el mundo académico a través de su crítica al pensamiento iluminista de los universales que presentaban como categoría dicotómica el binomio femenino-masculino, contribuyendo a la visualización de las relaciones asimétricas entre varones y mujeres y ampliando, o cuestionando, la definición biologicista del ser.

Gender no es sinónimo de mujer-mujeres, como muchos análisis traducen o vulgarizan, sino todo lo contrario; esta pensada como categoría para bucear en el complejo entramado de relaciones varón/mujer y dar a conocer, explicitar, poner en cuestión los mecanismos y los supuestos por los cuales a lo largo de la

\footnotetext{
${ }^{2}$ En especial debido a algunas convocatorias a crear archivos de mujeres. Este análisis puede hacerse extensivo a cualquier planteo de creación de archivos temáticos, dejando de lado los archivos que recogen testimonios orales ya que los mismos poseen especificidades que no abordaremos en este trabajo.
}

208 
historia, y en cada una de las coyunturas concretas, la pareja o el divorcio masculino/femenino se construyeron.

En este sentido, los documentos para trabajar la historia desde una perspectiva de género ¿̇erían otros, diferentes de aquellos que utiliza la historia "en general"3?

Hay un cierto consenso metodológico en el sentido de que los documentos no varían, sino lo que cambian son las preguntas que le realizamos a esos documentos y cómo los ponemos al servicio de comprender y visibilizar los dispositivos de control y de desigualación ${ }^{4}$, así como también de construir otras experiencias de género ${ }^{5}$. Esto estaría representado en la ecuación viejas fuentes-nuevas metodologías.

\section{Nuevos documentos}

Otra posibilidad de indagación es agrupar físicamente una serie de documentos de o sobre mujeres creando un centro de documentación. Esto nos posibilita recuperar documentos dispersos que pueden ser utilizados como fuente para la historia.

Esta tarea se realiza en un presente determinado, con un criterio temático, respondiendo a una necesidad circunstancial. Tiene una serie de limitaciones; por un lado confunde sobre si la perspectiva de género se resume en estudiar mujeres, es decir, sino estaría anulando su faceta relacional y, por otro lado, es necesario establecer muy claramente los criterios de selección documental. Por ejemplo, si se crea un centro de documentación de mujeres con participación política ${ }^{6}$, ¿qué mujeres incluiríamos?, ¿qué representarían cada una de las elegidas? ¿cómo se asigna la identidad? ¿podrían ser éstas categorías estáticas?

\footnotetext{
${ }^{3}$ La historia ha estado tan circunscripta a la del varón hasta no hace demasiado tiempo que la historia de las mujeres puede considerarse tan general como la del otro sexo. No vamos a profundizar en el debate, dicho en términos muy simples, de si la historia de las mujeres o con perspectiva de género debe formar parte de la historia general o si no debemos contribuir a esta por verse viciada de androcentrismo.

${ }^{4}$ Como tantas veces ha mencionado Ana María Fernández, los mecanismos no son de desigualdad en términos genéricos sino de desigualación, ya que hay quien ejerce ese poder, no es un mecanismo natural, sin por esto caer tampoco en una teoría vulgar conspirativa. Ver Fernández, Ana María: La mujer de la ilusión, Piados, Buenos Aires, 1994.

${ }^{5}$ Como apunta Teresa de Lauretis donde la experiencia se construye y no solo donde es descubierta o develada. Ver de Lauretis, Alicia: Alicia ya no, Cátedra, Madrid, 1992.

${ }^{6}$ Evitándonos teorizar sobre el concepto mujer, es decir si existe o no un colectivo mujer.
} 
Otra posibilidad es rescatar fondos documentales de particulares, de asociaciones de mujeres o feministas, lo que permitiría mantener la documentación en su contexto de producción, brindándonos las mismas posibilidades de indagación que posee cualquier documento de archivo.

Las organizaciones de mujeres y feministas son parte del entramado social que en la búsqueda de sus ideales despliega distintas estrategias, posiciones y premisas para llevar a cabo sus objetivos. Así como los movimientos sociales, las organizaciones políticas, las agrupaciones identitarias o los nucleamientos barriales, en general no conservan de modo orgánico, y en muchos casos de ningún modo, las producciones de sus prácticas plasmadas en discursos, panfletos, performance, manifestaciones, seminarios, capacitaciones, charlas-debate, etc. Aquello que los movimientos no registran en documentos puede formar parte de otros archivos. Los feminismos pueden tener su historia en las casas particulares de sus militantes pero también puede encontrarse registrada por los servicios de inteligencia ${ }^{7}$.

No obstante, el problema central estaría en concluir o asignarle a estos fondos un valor en-sí para la mirada de género que puede y debe leerse en todas las manifestaciones políticas, económicas, sociales ya sean estatales o privadas, religiosas o laicas.

En este punto debemos, necesariamente, diferenciar las tareas y las producciones que podemos desarrollar como historiadores/as y como archivistas.

Si como historiadoras/es con perspectiva de género creamos centros de documentación orientados a nuestros fines presentes, lo que estaríamos legando a futuras generaciones serían relatos fragmentados, "de autor" sobre determinados hechos y -este es el problema fundamental- sobre determinados documentos seleccionados y procesados por nosotros/as desde nuestros paradigmas actuales. Cuestiones que hoy nos pueden parecer naturales pueden ser un invisible social que no está oculto sino naturalizado. Con esto decimos que si como historiadoras/es trabajamos seleccionando los documentos que daremos a la consulta podemos cometer, por lo menos, dos descuidos: el primero, no ver todas las estrategias de desigualación de género porque estamos inmersas en ese proceso y, el segundo, asignarles un significado excesivamente arbitrario.

\footnotetext{
${ }^{7}$ Tal es el caso de la Unión de Mujeres Argentinas (UMA) cuyos accionares registró la Dirección de Inteligencia de la Policía de la Provincia de Buenos Aires engrosando el Fondo DIPBA, cuya custodia actualmente pertenece a la Comisión Provincial por la Memoria.
}

210 
La tercera y última posibilidad es que, al creer necesario y prioritario estudiar las relaciones de género, fundemos un centro de investigación digitalizando determinados documentos a los que tenemos acceso y a eso lo denominemos "archivo de género". ¿Cuáles serían los signos, símbolos, palabras, que nos indican que un documento puede ser estudiado en su faceta relacional? Nos encontramos nuevamente con las limitaciones del centro de documentación, a las que les podemos sumar otros interrogantes.

¿Cuáles son los signos que significan las asimetrías entre varones y mujeres?

¿Somos nosotras/os quiénes debemos asignarlos? ¿O cada una/o en su investigación va a hacer el recorte teórico-metodológico? Estas y otras preguntas forman parte de un debate no concluido.

Una novedosa manera de escribir la historia no es una posibilidad contenida en un documento, la ecuación nuevos archivos-nuevas metodologías nos puede conducir a un callejón sin salida o, lo que es peor, a parir otra ecuación: nuevos archivos -vieja historia.

\section{La accesibilidad documental en la Argentina y nuestro rol como investigadores/as}

Los archivos están, más allá de que no podamos acceder a ellos, salvo casos de destrucción intencional por acción, omisión o simplemente desidia estatal. Llegamos a ellos como usuarios/as a través del tratamiento archivístico que puede habérseles dado, lo que permite su consulta pública. Este es el último eslabón de una cadena que comienza con la posibilidad de conservación física de los documentos y continúa con la existencia de repositorios, servicios y equipamiento en los archivos, la organización y el respeto de los fondos documentales, la asignación de medios económicos y de personal, la elaboración de instrumentos de descripción y auxiliares, la difusión (de archivos, documentos y descriptores), la posibilidad de realizar reprografías para evitar la excesiva manipulación de los originales, la existencia de un equipamiento adecuado para la lectura de documentos audiovisuales o informatizados, etc. Siempre y cuando no haya restricciones legales de acceso a los mismos en función de la necesidad de proteger la seguridad del Estado y sus relaciones multilaterales, el respeto a la vida privada (protección de los datos sensibles), la propiedad intelectual, el secreto industrial y comercial y el derecho a la propiedad privada de los dueños de archivos ${ }^{8}$.

\footnotetext{
${ }^{8}$ Duchein, Michel: Los obstáculos que se oponen al acceso, a la utilización y a la transferencia de información conservada en los Archivos: Un estudio del RAMP, Unesco, París, 1983.
} 
Por lo tanto, exceptuando las limitaciones legales, cuando estos pasos están dados, y el archivo es de acceso público la consulta a los documentos es democrática. No depende de la buena o mala voluntad de la persona encargada de custodiar los documentos.

Frente a este planteo se nos podría objetar que la situación de los archivos en la Argentina dista mucho de ser el ideal con el que cualquier investigador/a pretendería encontrarse a la hora de realizar su trabajo. Tomando cada uno de los eslabones mencionados anteriormente, nos podemos encontrar con limitaciones que van desde fondos documentales que se encuentran en situación de riesgo absoluto con respecto a su preservación física, una gran cantidad de repositorios abiertos a la consulta pública "discrecionalmente", desorganización de fondos documentales, falta de instrumentos de descripción que permitan acceder a los mismos, etc. A esto debemos sumarle que la normativa referida al acceso a la documentación no tiene en cuenta el valor histórico que pueden tener determinadas series documentales y, o bien clasifica la documentación sin mencionar en que instancias y plazos debiera ser desclasificada, o directamente recomienda la destrucción de cualquier tipo de documentación que contenga datos sensibles una vez que venció su valor administrativo ${ }^{9}$ (por ejemplo: la nueva regulación sobre protección de datos personales).

Ahora, frente a esta perspectiva... ¿qué hacemos como usuarios/as? ¿Qué medidas tomamos? ¿Quién es responsable de la situación?

El trabajo del/la científico/a social suele ser una tarea individual, especialmente en la instancia de búsqueda documental. Es en esta solitaria tarea que las y los investigadores se ven limitados por los problemas de archivo antes mencionados y apurados por los plazos que implican la entrega de trabajos, avances, informes, etc. De alguna manera esto lleva a que frente a la falta de accesibilidad a determinados documentos rara vez se promuevan acciones conjuntas de reclamo o denuncia social y más frecuentemente se "soporten" las trabas, problemas, etc. O se arregle con el encargado/a del archivo el acceso o la búsqueda. Una vez resuelto el problema específico, ya no nos preocupa como investigadores/as lo que suceda con ese archivo, que en el mejor de los casos será que se mantenga en las mismas condiciones y con los mismos problemas. Esto genera un círculo vicioso del que difícilmente podamos salir. Este mecanismo

\footnotetext{
${ }^{9}$ Para un estudio de la clasificación de documentos secretos en la Argentina véase Nazar, M.: "La accesibilidad documental y sus limitaciones legales: los documentos secretos en la Argentina", ponencia presentada en el V Congreso de Archivología del Mercosur (Córdoba, 2003), edición en CD-ROM o en www.farargentina.com.ar
}

212 
no se altera necesariamente cuando algún/a científico/a social sin conocimientos específicos se transforma en responsable institucional de un archivo: mientras puedan tener acceso a los documentos que son de interés para él/ella y su grupo de pares, la situación podrá mantenerse como está. De esto puede resultar que el archivo termine siendo un coto cerrado adonde sólo accedan quienes son amigos/as, o sea se afianzen las redes clientelares ya existentes, se tejan nuevas y el acceso democrático a la documentación termine siendo sólo un slogan.

Si bien no somos tan ingenuas como para plantear que el campo científico es una carrera abierta al talento ${ }^{10}$, nos parece que garantizar el acceso democrático a la información es una buena manera de empezar a desmontar algunas trampas de la producción intelectual.

La situación es, mientras se mantengan los silencios y para algunos/as los privilegios, que no vamos a poder salir de esta inercia.

Frente a este conjunto de problemas, sugerimos varias cosas. En principio, deberíamos intentar generar espacios de intercambio y discusión entre historiadores/as y archivistas para poder articular las demandas frente al Estado en materia de políticas de preservación del patrimonio documental. Esto incluye tanto la preservación como el ordenamiento, la clasificación, la descripción, la consulta pública y la difusión. En este sentido, una de las prioridades debiera ser la puesta en práctica de un Sistema Nacional de Archivos y la revalorización del Archivo General de la Nación como órgano rector nombrando en su Dirección, a través de concursos transparentes, personal capacitado para llevar adelante esa tarea.

Sólo estableciendo políticas de desarrollo archivístico podremos empezar a tejer las redes para asegurar la accesibilidad documental para todos y todas, rompiendo con el clientelismo y garantizando la salvaguarda del patrimonio documental.

Si el Estado se hiciera cargo de llevar adelante una política de preservación del patrimonio documental, deberían encontrarse accesibles los archivos de instituciones u organismos con funciones represivas donde podríamos analizar el sesgo de género como, por ejemplo, en el poder judicial, las cárceles, hospitales, neurosiquiátricos, escuelas. E, incluso, si hubiera un sistema nacional de archivos

\footnotetext{
${ }^{10}$ Seguimos en este sentido, ente otras, la línea que se encuentra en Bordieu Pierre: Intelectuales, política y poder, EudeBA, Buenos Aires, 1999.
} 
podrían incluirse los de la Iglesia (conventos y parroquias), sociedades de ayuda o socorros mutuos, sindicatos, organismos de derechos humanos, partidos políticos, movimientos sociales, bancos, etc.

Entendiendo que las relaciones de desigualación de género son parte de las relaciones sociales de reproducción y dominación, en sentido general deberían poder rastrearse en todo archivo. ${ }^{11}$

\section{De la indagación de las relaciones de género en los archivos}

Si seguimos el encadenamiento planteado al presentar la accesibilidad, el punto en el que la desigualdad de género se podría evidenciar en la tarea archivística es en la descripción (antes de la cual se requiere haber dado los pasos previos que son la preservación, el ordenamiento y clasificación). En este punto, se hace necesario el intercambio con los y las archivistas.

En la tarea de descripción documental, la archivística prescribe que en la misma se debe reflejar (en forma sucinta) el contenido de la información del documento. Si la relación de género permanece invisibilizada, es lógico pensar que lo mismo sucedería en el momento de la descripción documental. Frente a ello, una de las primeras cuestiones que se pueden destacar es señalar a las mujeres en la descripción general del documento. Indicar el sexo en la descripción no tiene el fin de reducir a una marca biológica los mecanismos o procesos de desigualación de género sino ser una primera contribución a la indagación. En la medida en que esta práctica sea sistemática en todos los archivos evidenciará para los futuros investigadores que lo no-dicho es parte de lo silenciado intencionalmente y no por la utilización de un leguaje genérico que incluiría en lo masculino a ambos sexos.

En segundo lugar, incluir en el auxiliar descriptivo denominado índice temático las referencias a las relaciones de género. Para poder llevar adelante esa tarea es necesario que las personas encargadas del trabajo en archivos conozcan este tema para poder resaltarlo.

Tomemos como ejemplo el caso del Fondo Documental "Ministerio del Interior. Expedientes secretos, confidenciales y reservados (1932 - 1983)" que se encuentra abierto a la consulta pública en el Departamento Archivo Intermedio (DAI) del Archivo General de la Nación.

\footnotetext{
${ }^{11}$ Desde los legajos de personal y sumarios realizados en cualquier fábrica o en los mismos servicios de policía, hasta en los fundamentos de la selección de personal, los reglamentos internos de cualquier institución, los testamentos, padrones, etc.
}

214 
Cuando se planificó la descripción del mismo, eligiendo para ello la forma documento por documento, se optó por diferenciar el sexo de las personas mencionadas en los expedientes, en especial en el caso de los y las expulsados/as por la Ley de Residencia y los y las detenidas bajo Poder Ejecutivo Nacional.

Entonces, en el campo de la descripción general del expediente en vez de decir: "Solicita la detención y expulsión del país de veinte personas" o "Se dispone la detención bajo Poder Ejecutivo Nacional de diez individuos" se especifica si se trata de varones o de mujeres.

Ahora, una vez asegurada la descripción general del documento teniendo en cuenta la terminología empleada por el mismo, las relaciones de dominación no asumidas en el documento se destacan en el índice temático. Un caso muy frecuente en el fondo mencionado es la figura contravencional "escándalo". Si bajo esta denominación encontramos abusos sexuales, éstos son mencionados en el índice. Otro caso es el de acoso sexual, término no utilizado en el período que abarca el fondo: por tanto en la descripción general del documento encontramos la mención a "eleva denuncia de secretaria a su jefe inmediato" y en el índice se nomina: acoso sexual.

\section{A modo de conclusión}

Las tareas de investigación son sustancialmente diferentes a las de poner a la consulta documentos para que ella pueda realizarse. Debido a que se ha presentando la gestión documental como uno de los desafíos de las nuevas generaciones de investigadores, en este artículo quisimos evidenciar las implicancias negativas que tiene confundir estas dos tareas.

La alta difusión de nuevas perspectivas historiográficas que trabajan sobre el pasado reciente, toman como fuente los testimonios y problematizan el concepto de memoria, posiblemente colaboren en la homologación conceptual de objetos y categorías tan diferentes como memoria, testimonio, documento, fuente, archivo, centro de documentación, reservorio de información, artefacto cultural, etc.

Particularizando sobre el caso de los estudios de género presentamos las posibilidades y limitaciones que pueden encontrarse al crear centros de documentación, recuperar fondos particulares o de asociaciones de mujeres y al indagar sobre estas relaciones en fondos documentales tradicionales. Destacando la existencia de una disciplina que viene trabajando sobre los archivos hace cien años y utiliza 
por tanto una terminología específica y principios propios, desarrollados a partir de una práctica y una teoría sobre los documentos de archivo, no pretendimos con esto caer en una defensa corporativa. Sino que intentamos propiciar un acercamiento entre la archivística y las ciencias sociales a fin de contribuir a preservar y volver accesible el patrimonio documental no sólo para los/as investigadores/as, sino para la ciudadanía en su conjunto.

Nos parece importante generar espacios de discusión e intercambio con los archivistas y los encargados de archivos difundiendo la perspectiva de género en las producciones historiográficas, de forma tal de tenerlos de aliadas/os a la hora de desinvisibilizar las relaciones de género que pueden encontrarse en los fondos documentales, tanto a la hora de recuperar archivos como en las tareas de descripción documental.

En este sentido, la búsqueda de soluciones alternativas frente a las políticas de ausencia estatal en términos de archivo, no debieran ser el único accionar o respuesta posible ya que, en algún punto, estarían legitimando esa ausencia y propiciando la privatización de los mismos.

La tarea pendiente que tenemos como investigadores/as es exigirle al Estado una eficiente política de preservación del patrimonio documental. Política que debiera contar con las competencias técnicas necesarias en su planificación, la elaboración de normas que trasciendan el corto plazo y la generación de mecanismos para velar por su cumplimiento. Esto garantizaría la preservación, ordenamiento, clasificación, descripción, consulta pública y difusión de los documentos de archivo. 